\title{
Molecular Photoswitching Aided by Excited-State Aromaticity
}

Bo Durbeej, J un Wang and Baswanth Oruganti

The self-archived postprint version of this journal article is available at Linköping University Institutional Repository (DiVA):

http:// urn.kb.se/ resolve?urn=urn:nbn:se:liu:diva- 151723

N.B.: When citing this work, cite the original publication.

Durbeej, Bo, Wang, J ., Oruganti, B., (2018), Molecular Photoswitching Aided by Excited-State Aromaticity, ChemPlusChem, 83(11), 958-967. https:// doi.org/ 10.1002/ cplu.201800307

Original publication available at:

https:// doi.org/10.1002/ cplu.201800307

Copyright: Wiley (12 months)

http:// eu.wiley.com/WileyCDA/ 


\section{Molecular Photoswitching Aided by Excited-State Aromaticity}

Bo Durbeej, ${ }^{*[a]}$ Jun Wang, ${ }^{[a]}$ and Baswanth Oruganti ${ }^{[a, b]}$

[a] Assoc. Prof. B. Durbeej, Dr. J. Wang, Dr. B. Oruganti

Division of Theoretical Chemistry, IFM

Linköping University

SE-581 83 Linköping (Sweden)

E-mail:bodur@ifm.liu.se

[b] Dr. B. Oruganti

Department of Chemistry, GITAM Institute of Science (GIS)

GITAM University

Visakhapatnam-530045, Andhra Pradesh (India) 


\begin{abstract}
Central to the development of optoelectronic devices is the availability of efficient synthetic molecular photoswitches, the design of which is an arena where the evolving concept of excitedstate aromaticity (ESA) is yet to make a big impact. The aim of this minireview is to illustrate the potential of this concept to become a key tool for the future design of photoswitches. The paper starts with a discussion of challenges facing the use of photoswitches for applications and continues with an account of how the ESA concept has progressed since its inception. Then, following some brief remarks on computational modeling of photoswitches and ESA, the paper describes two different approaches to improve the quantum yields and response times of switches driven by $E / Z$ photoisomerization or photoinduced $\mathrm{H}$-atom/proton transfer reactions through simple ESA considerations. It is our hope that these approaches, verified by quantum chemical calculations and molecular dynamics simulations, will help stimulate the application of the ESA concept as a general tool for designing more efficient photoswitches and other functional molecules used in optoelectronic devices.
\end{abstract}




\section{Introduction}

The availability of synthetic molecular switches that can be controlled through light irradiation is central to the development of optoelectronic devices. ${ }^{[1-4]}$ A desirable feature of such photoswitches is photochromism, whereby the switches exist reversibly in two stable states with different electronic properties (e.g., HOMO-LUMO gaps, dipole moments and $\pi$-conjugation) and photochemical processes are employed to change the populations of the states. In this way, light can be used to control the macroscopic electronic properties (e.g., conductance) of the materials into which the switches are incorporated. Commonly, although only one of the two directions of interconversion between the states needs to be driven by light for the mechanism to be termed photochromic, this behavior is realizable by molecules that undergo reversible double-bond $E / Z$ photoisomerization ${ }^{[5-8]}$ or photocyclization ${ }^{[9-15]}$ reactions. Alternatively, the photochromic mechanism can involve reversible photoinduced electron ${ }^{[16]}$ or $\mathrm{H}$-atom/proton ${ }^{[17-19]}$ transfer reactions. Furthermore, there are also synthetic molecular switches where the interconversion between the two states can be triggered by both light and other stimuli (such as $\mathrm{pH}^{[12]}$ ), or is triggered by other stimuli than light altogether, such as temperature, $\mathrm{pH}$ or electric fields. ${ }^{[20]}$ Among the plethora of different classes of useful switches available today, Figure 1 depicts some operated through $E / Z$ photoisomerization, photocyclization or photoinduced $\mathrm{H}$-atom/proton transfer reactions, including azobenzene switches that tune the conductance of organic semiconductors, ${ }^{[3]}$ spiropyran switches that enable the fabrication of organic optical memory devices ${ }^{[1]}$ and salicylideneaniline switches that also can be driven by heat. ${ }^{[18]}$ 
- ElZ photoisomerization -

Stilbenes

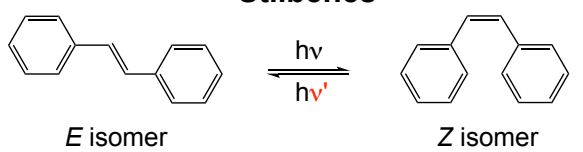

Azobenzenes

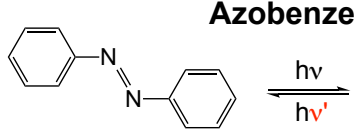

$E$ isomer

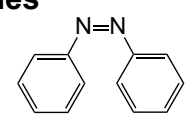

$\mathrm{Z}$ isomer

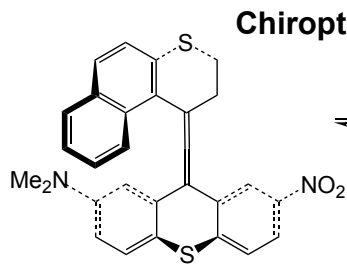

(P)-E isomer

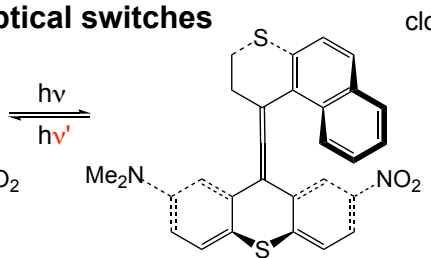

(M)-Z isomer

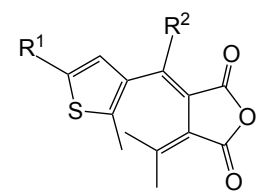

open isomer

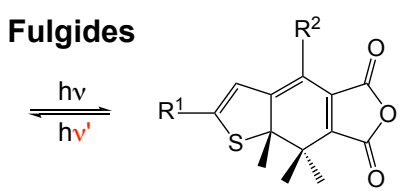

closed isomer

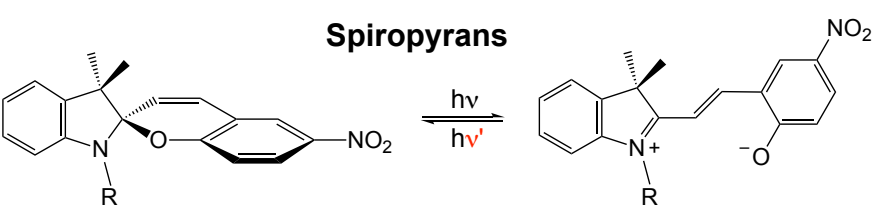

closed isomer open isomer

closed isomer

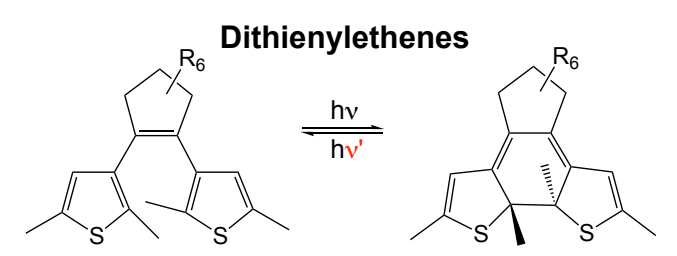

- Photoinduced H-atom/proton transfer -

Salicylideneanilines

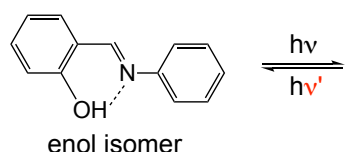

enol isomer

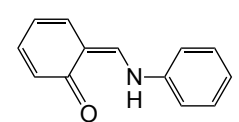

keto isomer

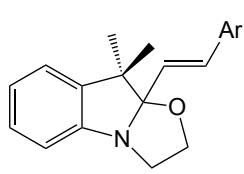

closed isomer
Oxazolidines

$\stackrel{\mathrm{hv}\left(\mathrm{or} \mathrm{H}^{+}\right)}{\stackrel{\left(\mathrm{orOH}^{-}\right)}{\longrightarrow}}$

(20)

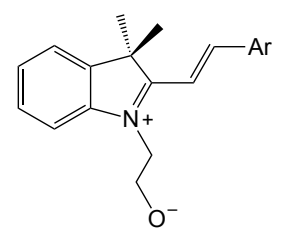

open isomer

Figure 1. Examples of different types of molecular switches powered by $E / Z$ photoisomerization, photocyclization or photoinduced $\mathrm{H}$-atom/proton transfer reactions.

Besides photochemical bistability, a number of additional factors are important for the application of molecular photoswitches. First, the switching from one of the states/isomers to the other should have a high quantum yield (QY) in both directions and also occur quickly so as to yield fast response times. In this connection, it is not surprising that photoisomerization is the basic governing mechanism for many of the synthetic photoswitches developed to date. Indeed, one of the most thoroughly studied biological switches - the visual pigment rhodopsin responsible for initiating dim-light vision in vertebrates $^{[21]}-$ relies on an ultrafast $\left(\begin{array}{lll}\sim 200 & \mathrm{fs}\end{array}\right)^{[22]} Z \rightarrow E$ photoisomerization for its function. Second, both isomers should be readily detectable, as a way to access or read the "information" associated with the respective state. This requires that the detection method (typically UV-Vis spectroscopy) does not trigger switching between the isomers - so-called non-destructive read-out. ${ }^{[23]}$ Notably, a key advantage of the chiroptical photoswitches (see Figure 
1) pioneered by Feringa and co-workers ${ }^{[6]}$ is that the distinct right-handed $(P)$ and left-handed $(M)$ helicities of the two isomers enable non-destructive read-out through monitoring of the optical rotation at wavelengths well separated from the wavelengths that induce switching. ${ }^{[23]}$ Alternatively, non-destructive read-out can be more easily realized by switches, such as the oxazolidine ones, ${ }^{[13]}$ that show large changes in a non-linear optical property (e.g., the first hyperpolarizability) between the isomers. Third, it is also important that the photoswitch can withstand numerous reaction cycles without concomitant degradation (fatigue resistance) ${ }^{[6]}$ and that its unique, intrinsic photochemical properties are retained as best as possible when it is incorporated into a device whose other components invariably perturb those properties. Finally, it is desirable to avoid having to trigger the switching with energetic UV light, ${ }^{[24]}$ which is more damaging to the device and its environment than visible or near-infrared light. However, since synthetic photoswitches are usually made up of rather small conjugated systems, this goal is difficult to reach.

In this minireview, we illustrate based on computational research how the performance of molecular photoswitches can be improved with respect to the first of the aforementioned criteria by invoking the evolving concept of excited-state aromaticity (ESA) ${ }^{[25,26]}$ First, developed as part of our own research on photoswitches more commonly known as light-driven rotary molecular motors (because their switching produces $360^{\circ}$ unidirectional rotary motion), ${ }^{[27-31]}$ we describe a novel approach using this concept for the design of $E / Z$ photoswitches with high QYs and fast response times. ${ }^{[30]}$ The discussion of this approach is followed by a presentation of another ESA-based strategy to achieve fast response times for photoswitches driven by $\mathrm{H}$-atom/proton transfer reactions, due to Rocha-Rinza and co-workers. ${ }^{[32]}$ By illustrating the merits of ESA for the design of photoswitches, we hope that the minireview will also help stimulate the application of this concept for the design of other functional molecules within optoelectronics.

\section{ESA}

Aromaticity is a central concept in organic chemistry and is widely employed to rationalize and predict structures and reactivities of cyclic conjugated compounds in the electronic ground state. Outside this realm, the concept was first applied to excited states in the 1960 s by Dewar ${ }^{[33]}$ and Zimmerman, ${ }^{[34]}$ who studied electrocyclic reactions and proposed that these, whether they occur thermally in the ground state or photochemically in an excited state, proceed via aromatic transition 
states. Subsequently, in 1972, Baird ${ }^{[35]}$ used molecular orbital theory (both qualitative arguments and semiempirical calculations) to predict rules for aromaticity and anti-aromaticity in the lowest triplet excited state $\left(\mathrm{T}_{1}\right)$ of cyclic conjugated hydrocarbons. Specifically, his analysis indicated that $4 n \pi$-electron systems $(n=0,1,2, \ldots)$ are aromatic and $(4 n+2) \pi$-electron systems are anti-aromatic in $T_{1}$. Notably, these rules, now known as Baird's rules, are the reverse of Hückel's $4 n+2$ and $4 n \pi$ electron rules for aromaticity and anti-aromaticity in the ground state $\left(\mathrm{S}_{0}\right)$. However, Baird's rules were not immediately put to use in studies of photochemical reactivity.

Much later, in 1998, support for Baird's results was presented by Gogonea, Schleyer and Schreiner, ${ }^{[36]}$ in the form of quantum chemical evidence that triplet states of $4 n \pi$-electron annulenes are aromatic rather than anti-aromatic. This work was followed by a renewed interest in ESA, ${ }^{[25,26,37-44]}$ particularly from Ottosson ${ }^{[25,26,38,39,43]}$ and Karadakov. ${ }^{[40,41,44]}$ For example, in 2008, Karadakov ${ }^{[40,41]}$ reported quantum chemical calculations showing that Baird's rules apply also to the lowest singlet excited state $\left(\mathrm{S}_{1}\right)$ of benzene and cyclooctatetraene. The $\mathrm{S}_{1}$ state of cyclobutadiene, in contrast, was found to follow Hückel's rules. ${ }^{[40,42]}$ As for the second singlet excited state $\left(\mathrm{S}_{2}\right)$, in turn, cyclobutadiene follows Baird's rules ${ }^{[42]}$ whereas benzene follows Hückel's rules. ${ }^{[44]}$ Thus, the computational results regarding the aromatic character of cyclobutadiene and benzene in different electronic states can be summarized as in Figure 2.

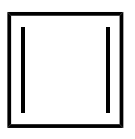

Cyclobutadiene

$\mathrm{S}_{0}$ anti-aromatic $\mathrm{T}_{1}$ aromatic

$\mathrm{S}_{1}$ anti-aromatic $\mathrm{S}_{2}$ aromatic

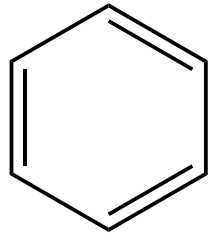

Benzene

$\mathrm{S}_{0}$ aromatic

$\mathrm{T}_{1}$ anti-aromatic

$\mathrm{S}_{1}$ anti-aromatic

$\mathrm{S}_{2}$ aromatic

Figure 2. The aromatic character of cyclobutadiene and benzene in different electronic states as predicted by quantum chemical calculations. ${ }^{[35,36,40-42,44]}$

The foregoing discussion makes it clear that, from a theoretical viewpoint, there is ample support for the occurrence of aromaticity reversal (relative to $\mathrm{S}_{0}$ ) in low-lying excited states. From 
an experimental viewpoint, spectroscopic evidence for this phenomenon has recently appeared in UV-Vis and IR studies of hexaphyrins by Kim and co-workers. ${ }^{[4-47]}$ Furthermore, direct structural evidence for the onset of ESA has been reported in studies of cyclooctatetraene-featuring molecules by electron diffractometry. ${ }^{[48]}$ However, despite ESA now being a well-established concept, its application to rationalize or tailor photochemical reactivity is still in an early stage, ${ }^{[30,32,49-52]}$ with one notable exception being its use by Aihara already in the 1970s to predict the allowedness of pericyclic reactions. ${ }^{[53]}$ Hence, we believe it is a very worthwhile goal of this minireview to illustrate the vast potential of ESA for the design of efficient molecular photoswitches.

\section{Some computational considerations}

Computational studies of photochemical reactions and ESA come with a number of challenges. In this section, we will briefly discuss two of them, which we believe are the main ones in the context of the present minireview.

The first challenge is that a reliable assessment of the efficiency (or QY) of a photochemical reaction demands both an accurate quantum chemical description of the excited state(s) involved as well as explicit modeling of the reaction dynamics. Given that many photochemical reactions (such as $E / Z$ photoisomerizations ${ }^{[54]}$ ) involve situations where an excited state is brought closer and closer to the ground state as the reactions proceed, it is often the case that expensive multi-reference quantum chemical methods like those based on complete active space self-consistent field (CASSCF) theory ${ }^{[55,56]}$ have to be used. Furthermore, although the possibility to propagate the nuclei with classical mechanics does make modeling of the reaction dynamics feasible, this comes at the prize of having to calculate numerous photoisomerization trajectories with different initial nuclear configurations and velocities, as a way to account for nuclear quantum effects in an indirect fashion. Also, there is a need to calculate the trajectories through non-adiabatic molecular dynamics (NAMD) simulations, ${ }^{[57-60]}$ so as to allow hops from one state to another depending on the energy gap and non-adiabatic coupling between the states. The NAMD results that we will present below were obtained by computing the non-adiabatic coupling with the algorithm developed by Robb and co-workers ${ }^{[57]}$ as implemented in the MOLCAS 8.0 suite of programs. ${ }^{[61]}$

The second challenge concerns the choice of approach to quantify the ESA. For this, several

calculable aromaticity indices are available, such as the Shannon aromaticity (SA) index, ${ }^{[62]}$ the 
harmonic oscillator model of aromaticity (HOMA) index, ${ }^{[63,64]}$ or the nucleus-independent chemical shift (NICS) index. ${ }^{[65]}$ Focusing, for the sake of brevity, here on the SA and HOMA indices used in our own work, SA is an electronic index based on Bader's theory of atoms in molecules. ${ }^{[66]}$ More precisely, this index probes the variation in electron density at bond critical points (BCPs) of the presumed aromatic ring and is formulated in terms of the Shannon entropy, ${ }^{[67]}$ defined as

$S=-\sum_{i}^{N} p_{i}\left(\mathbf{r}_{\mathrm{c}}\right) \ln p_{i}\left(\mathbf{r}_{\mathrm{c}}\right)$

Here, $\mathbf{r}_{\mathrm{c}}$ is a BCP, $N$ is the number of BCPs, and $p_{i}\left(\mathbf{r}_{\mathrm{c}}\right)$ is the normalized probability electron density at a given $\mathrm{BCP}$, defined as

$$
p_{i}\left(\mathbf{r}_{\mathrm{c}}\right)=\frac{\rho_{i}\left(\mathbf{r}_{\mathrm{c}}\right)}{\sum_{i}^{N} \rho_{i}\left(\mathbf{r}_{\mathrm{c}}\right)},
$$

where $\rho_{i}\left(\mathbf{r}_{\mathrm{c}}\right)$ is the electron density at that BCP. With these definitions, the SA index is obtained as the difference between the Shannon entropy that the system under investigation would have if it was perfectly aromatic and the Shannon entropy that it actual has. Thereby, a small SA value indicates that the variation in electron density at BCPs is small, as expected for an aromatic system. ${ }^{[62]}$ As a rough rule of thumb, aromatic compounds show SA values below 0.003. ${ }^{[62]}$

HOMA, finally, is a geometric index based on the deviation of the carbon-carbon bond lengths $R_{i}$ of the presumed aromatic ring from an ideal aromatic reference bond length $R_{\text {opt }}{ }^{[63,64]}$ which is taken to be that of benzene. Specifically,

HOMA $=1-\frac{\alpha}{n} \sum_{i}^{n}\left(R_{i}-R_{\mathrm{opt}}\right)^{2}$,

where $n$ is the number of carbon-carbon bonds and $\alpha$ is an empirical normalization factor chosen such that the HOMA value approaches 1 for an aromatic compound with all $R_{i}$ close to $R_{\mathrm{opt}}$, and approaches 0 for the corresponding non-aromatic Kekulé structures. ${ }^{[63,64]}$ 


\section{Photoswitching aided by ESA: $E / Z$ switches}

In this section, we will describe our recently discovered strategy to exploit ESA for the design of $E / Z$ photoswitches with high QYs and fast response times. ${ }^{[30]}$ The background is as follows. Namely, through NAMD simulations of light-driven molecular motors that utilize $E / Z$ photoisomerizations to produce unidirectional rotary motion, it has been found that a viable approach to extract high QYs from such motors is to incorporate in them a protonated or alkylated nitrogen Schiff base (see Figure 3). ${ }^{[29,68]}$ This approach relies on the electron-withdrawing ability of the cationic nitrogen center to promote heterolytic rather than homolytic cleavage of the $\pi$-bond of the isomerizing carbon-carbon double bond. Thereby, the rotary motion becomes less disturbed by pyramidalization of these carbons and can proceed in an efficient axial (rather than precessional) fashion (see Figure 3). ${ }^{[69]}$

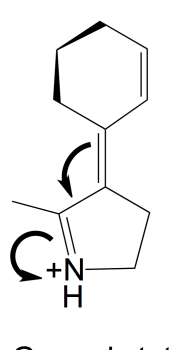

Ground state

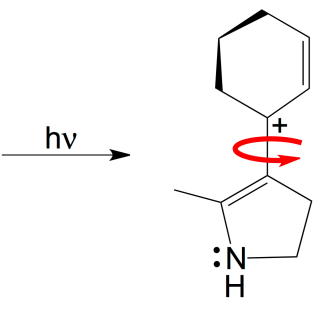

Excited state

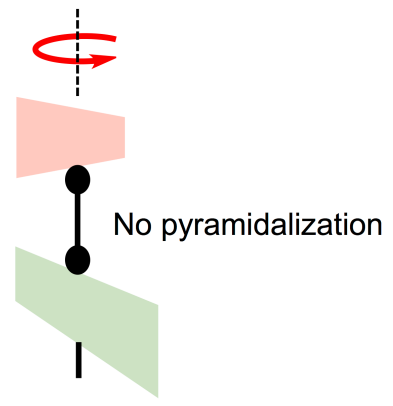

Axial rotary motion

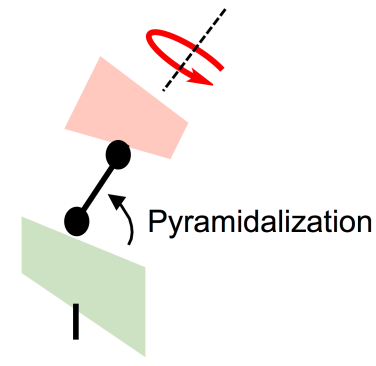

Precessional rotary motion

Figure 3. Photoinduced heterolytic $\pi$-bond cleavage of the isomerizing bond in a Schiff-base molecular motor and comparison of axial and precessional rotary motion. Adapted with permission from Ref. [69]. Copyright 2014, American Chemical Society.

Against this background, it is clearly of interest for the design of efficient $E / Z$ photoswitches to develop new chemical strategies that can replicate the behavior of Schiff-base motors, but without making use of a cationic nitrogen center to trigger heterolytic $\pi$-bond cleavage. Pleasingly, one such strategy is afforded by ESA. The underlying idea, exemplified by molecule 1 in Figure 4, is to favor a heterolytic process by allowing one of the rings connected to the isomerizing olefinic bond to become aromatic in the excited state through electron donation from the other ring. To this end, 1 features a cyclopentadiene motif, which is not aromatic but can be transformed into a 
cyclopentadienyl anion (which is well known to be aromatic) in the bright $S_{2}$ state of $\mathbf{1}$ through electron donation from a $\mathrm{N}$-methylpyrrolidine motif. The computational assessment of this idea, ${ }^{[30]}$ which we turn to next, was done using molecule $\mathbf{2}$ in Figure 4 as reference system. As can be seen, $\mathbf{2}$ is an isoelectronic analogue of $\mathbf{1}$ where the cyclopentadiene motif is replaced by cyclopentene. Hence, ESA is not possible in 2.

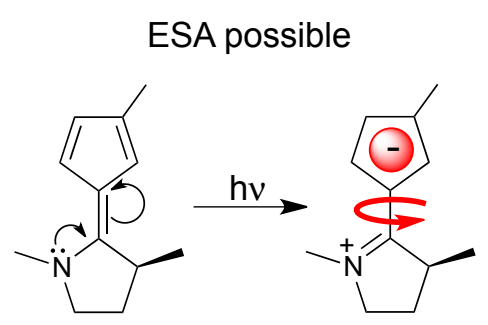

$1-E$

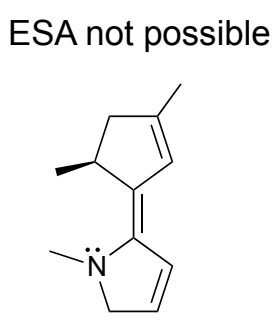

2-E

Figure 4. Chemical structures of molecules 1 and $\mathbf{2}$ shown in the $E$ isomeric forms with respect to the central olefinic bond, and the manifestation of ESA in the $\mathrm{S}_{2}$ state of 1.

First, the UV-induced $E / Z$ photoisomerizations of $\mathbf{1}$ and $\mathbf{2}$ were modeled by performing CASSCF minimum energy path (MEP) calculations starting from the vertically excited FranckCondon (FC) points in the respective bright state $-S_{2}$ for $\mathbf{1}$ and $S_{1}$ for $\mathbf{2}$, as predicted by calculations with the complete active space state-interaction (CASSI) and complete active space second-order perturbation theory (CASPT2) methods. ${ }^{[56,70]}$ From the MEP results summarized in Figure 5, which give an unbiased description of how the systems respond to light absorption without enforcing a pre-defined reaction coordinate, it is clear that the geometric evolution in the $\mathrm{S}_{2}$ state of $\mathbf{1}$ and the $S_{1}$ state of $\mathbf{2}$ is dominated by barrierless $E / Z$ torsional motion around the central olefinic bond. Thus, even though neither 1 nor 2 would qualify as a particularly good $E / Z$ photoswitch (i.e., for both molecules the electronic features of the $E$ and $Z$ isomers are too similar), they are proper models for investigating how ESA might influence $E / Z$ photoisomerization. This is also confirmed by the fact that starting CASSCF $\mathrm{S}_{0}$ geometry optimizations from the end points of the MEPs for the $E$ isomers yields the $Z$ isomers, and vice versa. ${ }^{[30]}$ 

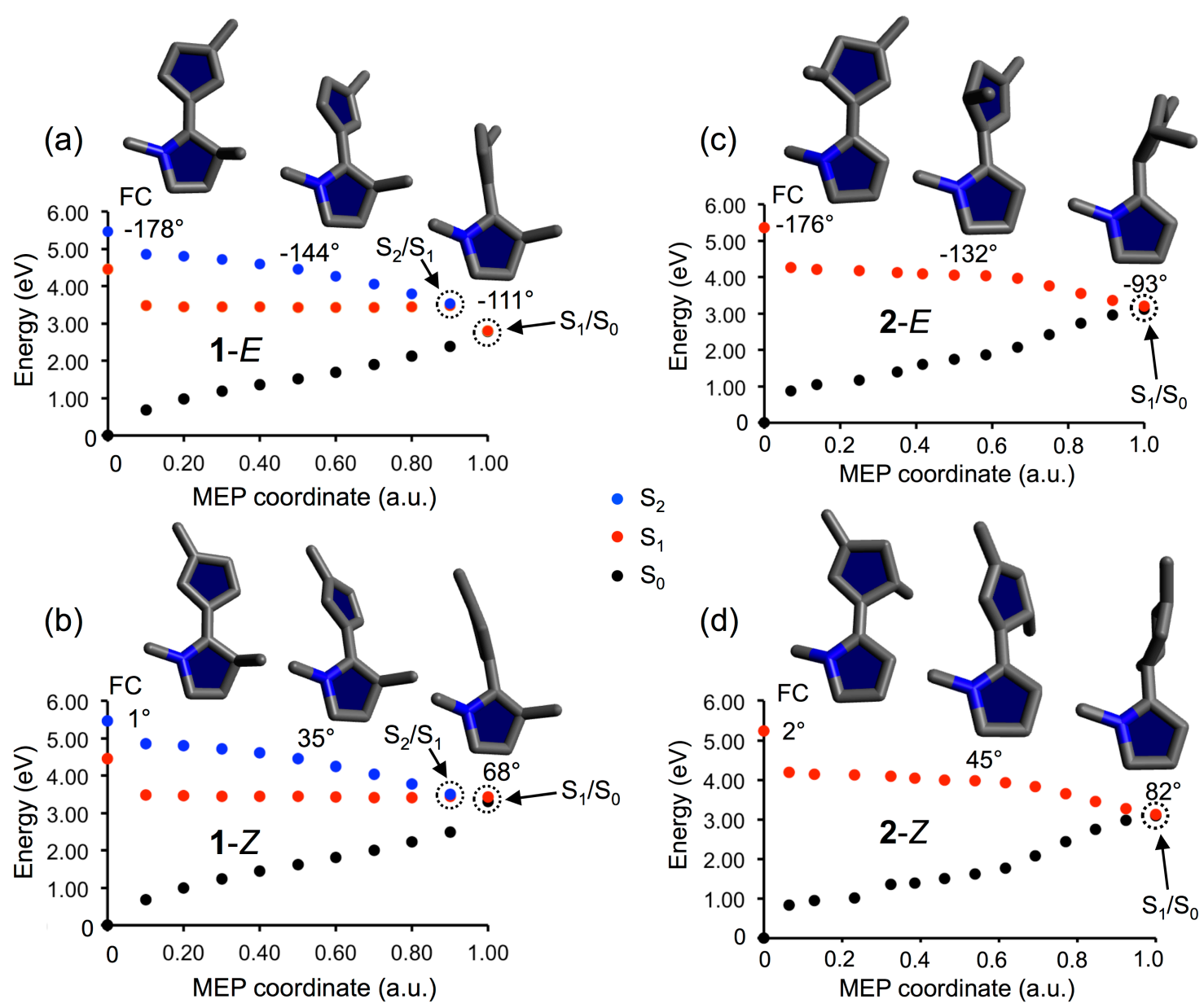

- $\mathrm{S}_{2}$

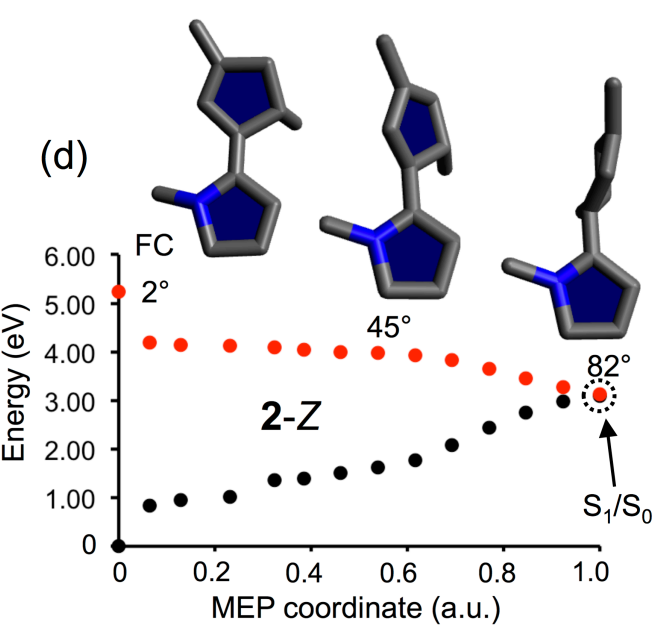

Figure 5. CASSCF MEPs from the FC points of the $E$ (a) and $Z$ (b) isomers of 1 and of the $E$ (c) and $Z(\mathrm{~d})$ isomers of 2 . All calculations carried out with the cc-pVTZ basis set and with active spaces comprising eight electrons ( $\operatorname{six} \pi$ and the nitrogen lone pair) distributed in seven orbitals. Shown are also selected molecular geometries along the paths, as well as the corresponding isomerization dihedral angles. Encircled points are close to assumed conical intersection (CI) regions where the systems can decay from the upper state to the lower. Notably, upon decay to the $\mathrm{S}_{1}$ state through a $\mathrm{S}_{2} / \mathrm{S}_{1} \mathrm{CI}, 1$ reaches a $\mathrm{S}_{1} / \mathrm{S}_{0} \mathrm{CI}$ with little further geometric distortion. Adapted with permission from Ref. [30]. Copyright 2017, American Chemical Society.

In order to quantitatively assess the effect of ESA on the photochemical efficiency, the photoisomerizations were then modeled by means of NAMD simulations, again starting from the FC points in the respective bright state $\left(S_{2}\right.$ for $\mathbf{1}$ and $S_{1}$ for 2$)$. The simulations were run at the CASSCF level for maximally 800 fs and with 200 different initial nuclear configurations and velocities for both the $E$ and $Z$ isomers of $\mathbf{1}$ and 2. Defining a successful photoisomerization trajectory as one that completes a net $180^{\circ}$ rotation around the central olefinic bond within $800 \mathrm{fs}$, 
the percentage of successful trajectories among the 200 can be taken as a measure of the corresponding QY. Furthermore, trajectory-averages of the time it takes to first reach the $\mathrm{S}_{0}$ state and the time needed to complete a net $180^{\circ}$ rotation give estimates of the excited-state lifetime $\langle\tau\rangle$ and photoisomerization time $<$ PIT $>$ of each process. All of these results are presented in Figure 6, alongside the distributions of $\tau$ and PIT values for individual trajectories.
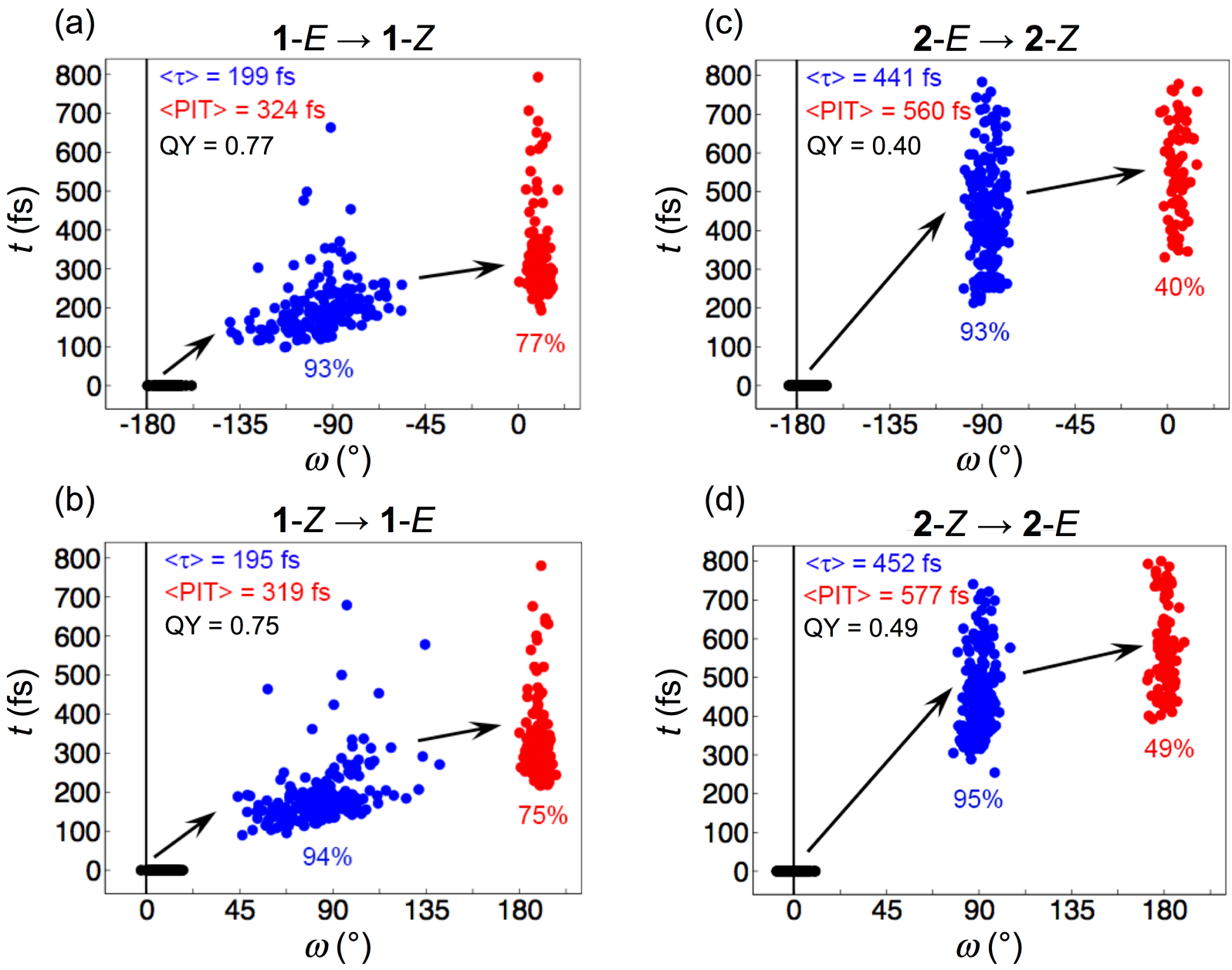

Figure 6. CASSCF NAMD trajectories from the FC points of the $E$ (a) and $Z$ (b) isomers of 1 and of the $E$ (c) and $Z$ (d) isomers of 2 . All calculations carried out with the 6-31G(d) basis set and with active spaces comprising eight electrons (six $\pi$ and the nitrogen lone pair) distributed in seven orbitals. The distributions of $\tau$ and PIT values are shown in blue and red fonts, respectively, alongside the corresponding changes in the isomerization dihedral angle $(\omega)$ relative to the starting nuclear configurations (in black font). Adapted with permission from Ref. [30]. Copyright 2017, American Chemical Society. 
As can be deduced from Figure 6, the presumed ESA of 1 has a major positive effect on the QYs and $\langle\tau>$ and $<$ PIT $>$ values shown by this molecule. Specifically, the QYs of 1 are not only high (0.75 and 0.77$)$, but are also markedly higher than the QYs of 0.40 and 0.49 achieved by 2 , which we know cannot exhibit ESA. In a similar vein, the $\langle\tau\rangle$ and $\langle$ PIT $>$ values of 1 are small both in an absolute sense (only $\sim 200$ and $\sim 320 \mathrm{fs}$ ) and relative to 2 , for which they amount to $\sim 450$ and $\sim 570 \mathrm{fs}$, respectively. Interestingly, even though 1 lacks the ability of Schiff-base molecules to facilitate $E / Z$ photoisomerizations through the presence of a cationic center, ${ }^{[69]}$ the photochemical performance of 1 compares very favorably with that of Schiff-base molecules as predicted by analogous NAMD simulations. ${ }^{[29,68]}$ Overall, then, Figure 6 highlights the vast unexplored potential of ESA to facilitate the future development of $E / Z$ photoswitches with high QYs and fast response times.

The above-described investigation also provided direct evidence that the cyclopentadiene motif of 1 does indeed become aromatic in the $S_{2}$ state of 1 by attaining the character of a cyclopentadienyl anion, as hitherto merely assumed. ${ }^{\left[{ }^{[0]}\right.}$ This evidence, in the form of electronic SA and geometric HOMA indices calculated from CASSCF wave functions and geometries along the photoisomerization MEPs of $\mathbf{1}$, is shown in Figure 7. Notably, the SA values become very small $(0.0001-0.001)$ as the reactions proceed, compared to the situation at the $\mathrm{S}_{2}$ FC points $(\sim 0.007)$. Indeed, SA values of such small magnitudes are perfectly consistent with an aromatic system. ${ }^{[62]}$ The same conclusion can be drawn from the HOMA values, which are $\sim 0$ at the $\mathrm{S}_{2} \mathrm{FC}$ points and then approach $\sim 0.8-$ a value well within the range considered to reflect aromaticity. ${ }^{[63,64]}$ Furthermore, NICS values that were also calculated (albeit less comprehensively so) reveal a gain in aromaticity during the photoisomerizations also based on widely used ${ }^{[65,71,72]}$ magnetic criteria. ${ }^{[30]}$ As outlined in Ref. [30], the reason why less focus was put on NICS values than on SA and HOMA values in the analysis is technical. Namely, while the MEPs needed to be calculated with stateaveraging over the $\mathrm{S}_{0}, \mathrm{~S}_{1}$ and $\mathrm{S}_{2}$ states in the CASSCF treatment, NICS values are more easily obtained from state-specific CASSCF wave functions. However, such wave functions are difficult to converge as the states come closer in energy as the photoisomerizations proceed. 

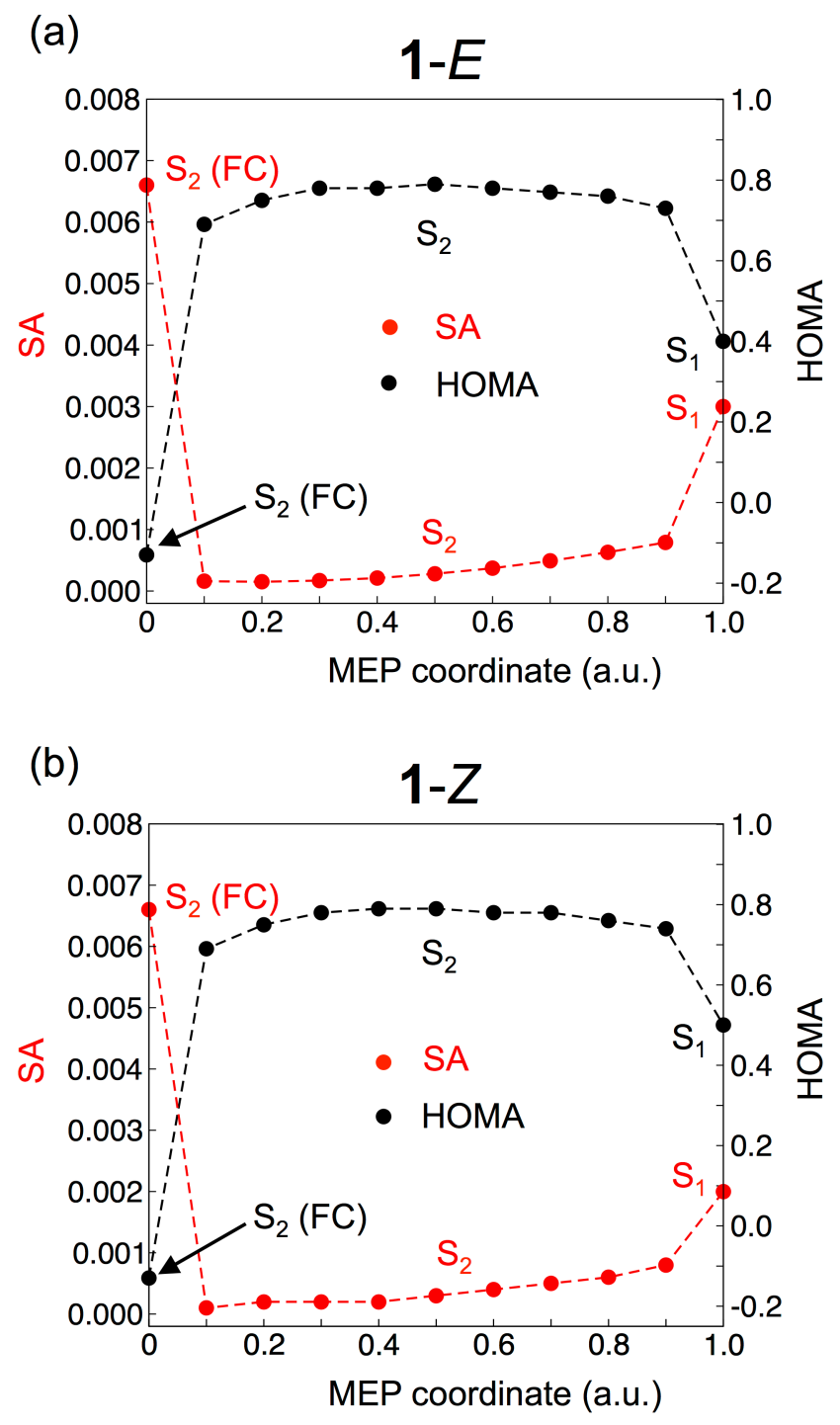

Figure 7. SA and HOMA values for the cyclopentadiene motif along the photoisomerization MEPs of the $E$ (a) and $Z$ (b) isomers of 1. Adapted with permission from Ref. [30]. Copyright 2017, American Chemical Society.

The fact that the SA and HOMA values offer a consistent picture of the onset of aromaticity in the $\mathrm{S}_{2}$ state of $\mathbf{1}$ is particularly pleasing given that no single aromaticity index is universally applicable and different indices are not always correlated. ${ }^{[73-76]}$ For example, the HOMA index is not well suited to probe the aromaticity of transition states for pericyclic reactions. ${ }^{[77]}$ Furthermore, of more direct relevance for this work, caution must be exercised when using HOMA to describe ESA, as the $R_{\text {opt }}$ parameter in Eq. 3 refers to the ground state. However, the above HOMA-based assessment of changes in aromaticity in an excited state should not be marred by this problem. 


\section{Photoswitching aided by ESA: H-atom/proton transfer switches}

While the previous section highlights a route to improve the performance of $E / Z$ photoswitches by the onset of aromaticity in an excited state, Rocha-Rinza ${ }^{[32]}$ and co-workers have nicely demonstrated how the performance of photoswitches involving $\mathrm{H}$-atom/proton transfer reactions conversely is helped by the loss of aromaticity upon photoexcitation. Specifically, these researchers set out to identify the driving force responsible for the ultrafast $\left(50 \mathrm{fs}^{[78]}\right)$ excited-state intramolecular proton transfer (ESIPT) of salicylideneaniline that, as shown in Figure 8a, converts the more stable enol isomer ${ }^{[79]}$ to the keto isomer and is a key step for the function of salicylideneaniline photoswitches. ${ }^{[17,18]}$ Clearly, this proton transfer is expected to be difficult to achieve in the $\mathrm{S}_{0}$ state, as the aromatic phenol moiety is transformed into a cyclohexadienone motif. But what about the situation in the photoactive $S_{1}$ state? To address this question, these authors first used density functional theory (DFT) and time-dependent DFT (TD-DFT) at the level of the $\tau \mathrm{HCTH}$-hyb functional ${ }^{[80]}$ to calculate potential energy curves for the enol-keto tautomerization of salicylideneaniline in the $\mathrm{S}_{0}$ and $\mathrm{S}_{1}$ states, along a reaction coordinate where the distance between the phenol oxygen and the transferred proton $\left(d_{\mathrm{O}-\mathrm{H}}\right)$ increases. As can be seen from Figure $8 \mathrm{~b}$, these calculations predict markedly different energy profiles in the two states; uphill in $\mathrm{S}_{0}$ through a barrier of about $5 \mathrm{kcal} \mathrm{mol}^{-1}$ and downhill in $\mathrm{S}_{1}$ through no barrier at all.

(a)

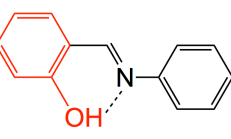

enol isomer aromatic
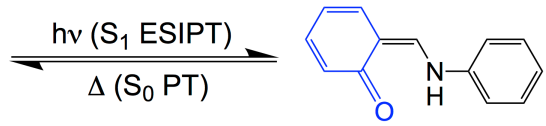

keto isomer non-aromatic (b)

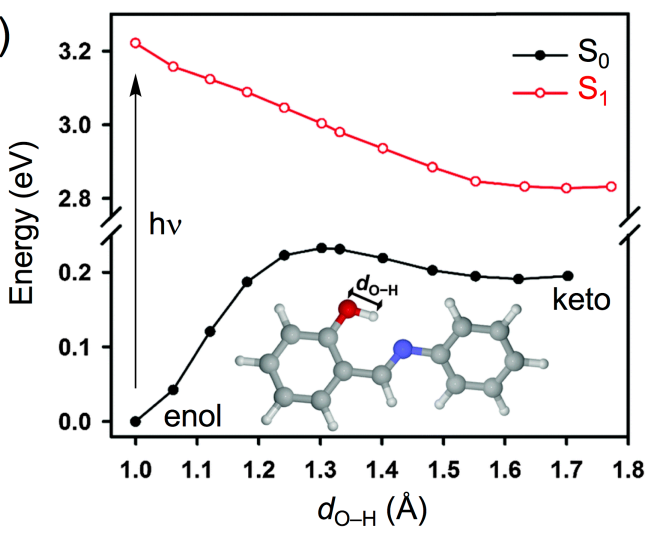

Figure 8. (a) Excited-state intramolecular proton transfer in salicylideneaniline. (b) $\mathrm{S}_{0}$ and $\mathrm{S}_{1}$ potential energy curves for the enol-keto tautomerization of salicylideneaniline calculated at the $\tau \mathrm{HCTH}-\mathrm{hyb} / 6-311 \mathrm{G}++\mathrm{G}(\mathrm{d}, \mathrm{p})$ level of theory. Adapted from Ref. [32] with permission from the PCCP Owner Societies. 
Next, Rocha-Rinza and co-workers ${ }^{[32]}$ proceeded to rationalize these results by means of atoms in molecules techniques based on TD-DFT that allow electron densities to be assigned to individual atoms also in excited states. ${ }^{[81]}$ Specifically, of particular interest are the changes in aromatic character of the $\mathrm{C}_{6} \mathrm{H}_{4}$ fragment (see Figure 9) in the $\mathrm{S}_{0}$ and $\mathrm{S}_{1}$ states as the enol isomer is converted to the keto isomer (and the bonding in the $\mathrm{C}_{6} \mathrm{H}_{4}$ fragment changes). Employing an electronic aromaticity index that probes how well the carbon electrons of the $\mathrm{C}_{6} \mathrm{H}_{4}$ fragment are delocalized in the $\mathrm{S}_{0}$ and $\mathrm{S}_{1}$ states of salicylideneaniline compared to the carbon electrons in the $\mathrm{S}_{0}$ state of benzene, ${ }^{[82]}$ the results of this analysis are presented in Figure 9.

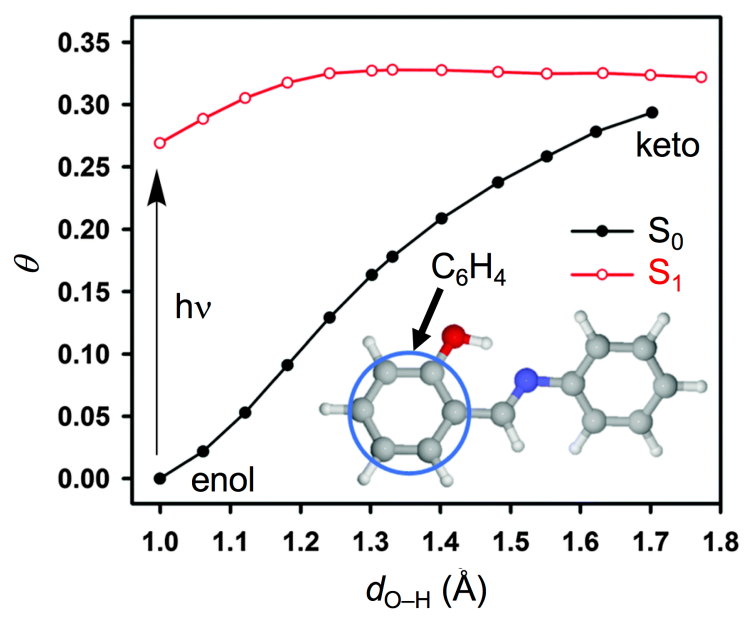

Figure 9. Aromaticity index $\theta$ defined in Ref. [82] for the $\mathrm{C}_{6} \mathrm{H}_{4}$ fragment along the $\mathrm{S}_{0}$ and $\mathrm{S}_{1}$ potential energy curves for the enol-keto tautomerization of salicylideneaniline. Adapted from Ref. [32] with permission from the PCCP Owner Societies.

Notably, with the index defined in such a way that it amounts to 0 for $\mathrm{S}_{0}$ benzene and increases with reduced aromatic character, ${ }^{[82]}$ in both states the $\mathrm{C}_{6} \mathrm{H}_{4}$ fragment loses aromaticity as a result of the enol-keto tautomerization. However, whereas the loss is gradual in $\mathrm{S}_{0}$, in $\mathrm{S}_{1}$ almost all of the aromaticity that is lost is lost already upon light absorption of the parent enol isomer. This means that the subsequent ESIPT to form the keto isomer is not at all hindered like the proton transfer in the ground state is. In fact, these results suggest that the ESIPT is facilitated and made barrierless by the considerable loss of aromaticity from the initial photoexcitation. Thus, for the design of molecular switches that utilize a photoinduced $\mathrm{H}$-atom/proton transfer reaction to convert an aromatic isomer into a non-aromatic one (but utilize heat for the reverse process, see Figure 8a), 
fast response times appear achievable by ensuring that the aromatic character is substantially weakened upon light absorption.

Finally, it should be mentioned that the above idea was probably first put forth by Ottosson and co-workers, ${ }^{[49]}$ who suggested - but without providing any direct proof of fast photoswitching to use photoinduced $\mathrm{H}$-atom/proton transfer reactions for the construction of molecular conductance switches that incorporate a central aromatic benzene moiety as shown in Figure 10. Designating the isomers as "OFF" and "ON", a key distinguishing feature between the two species is the occurrence of a saturated $\mathrm{sp}^{3} \mathrm{C}$-atom at the benzylic position of the OFF species that breaks the $\pi$-conjugation and limits the conductance. It was hypothesized that the OFF/ON photoswitching that involves conversion of this $\mathrm{sp}^{3} \mathrm{C}$-atom to an $\mathrm{sp}^{2} \mathrm{C}$-atom is facilitated by a loss of aromaticity in the central benzene moiety upon photoexcitation. ${ }^{[49]}$

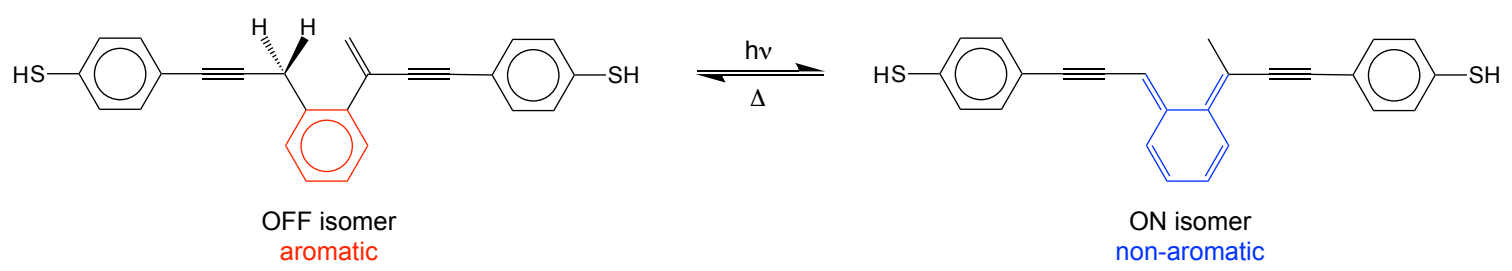

Figure 10. Molecular conductance switch proposed by Ottosson and co-workers. ${ }^{[49]}$ The central aromatic benzene moiety (in red font) of the OFF isomer is transformed into a non-aromatic orthoxylylene motif (in blue font) in the ON isomer.

\section{Summary and Outlook}

In view of the need to develop more efficient and faster molecular photoswitches that can enhance the performance of optoelectronic devices, we have in this minireview outlined two different approaches to reach this goal that invoke the ESA concept in fundamentally opposite ways. The first approach concerns the design of $E / Z$ photoswitches and is based on allowing a moiety adjacent to the isomerizing double bond to become aromatic in the photoactive excited state. The potency of this strategy is discussed in light of recent NAMD simulations performed to compare the photoisomerization efficiency of two molecules with and without a moiety behaving in this way. ${ }^{[30]}$ Specifically, we describe how these simulations predict that the strategy holds great promise in improving both the QYs and response times of $E / Z$ photoswitches. The second approach, in turn, is 
due to work by Rocha-Rinza ${ }^{[32]}$ and co-workers and applies to the design of switches powered by photoinduced $\mathrm{H}$-atom/proton transfer reactions. Here, conversely, the idea is to facilitate the switching by rather allowing an aromatic donor moiety to lose aromaticity by photoexcitation, whereby it becomes more reactive and the transfer process is made fast and barrierless.

In closing, we emphasize that the potential of using ESA as a photochemical design tool is heavily unexplored. Therefore, given the positive results reported in this minireview and the proven merits of computational modeling in studies of molecular photoswitches, ${ }^{[83-87]}$ it appears worthwhile for future research to investigate whether the ESA concept also can be employed to improve molecular photoswitches in other aspects than those discussed herein.

\section{Acknowledgements}

We acknowledge financial support from the Swedish Research Council (grant 621-2011-4353), the Olle Engkvist Foundation (grants 2014/734 and 184-568), the Carl Trygger Foundation (grant CTS 15:134) and Linköping University, as well as grants of computing time at the National Supercomputer Centre (NSC) in Linköping.

\section{Conflict of Interest}

The authors declare no conflict of interest.

\section{Keywords}

aromaticity $\bullet$ hydrogen transfer $\bullet$ isomerization $\bullet$ molecular electronics $\bullet$ photochromism 


\section{References}

[1] H. Zhang, X. Guo, J. Hui, S. Hu, W. Xu, D. Zhu, Nano Lett. 2011, 11, 4939-4946.

[2] E. S. Tam, J. J. Parks, W. W. Shum, Y.-W. Zhong, M. B. Santiago-Berríos, X. Zheng, W. Yang,

G. K.-L. Chan, H. D. Abruña, D. C. Ralph, ACS Nano 2011, 5, 5115-5123.

[3] E. Orgiu, P. Samorì, Adv. Mater. 2014, 26, 1827-1845.

[4] Z. Liu, S. Ren, X. Guo, Top. Curr. Chem. 2017, 375, 56.

[5] D. H. Waldeck, Chem. Rev. 1991, 91, 415-436.

[6] B. L. Feringa, R. A. van Delden, N. Koumura, E. M. Geertsema, Chem. Rev. 2000, 100, 17891816.

[7] H. M. D. Bandara, S. C. Burdette, Chem. Soc. Rev. 2012, 41, 1809-1825.

[8] X. Guo, J. Zhou, M. A. Siegler, A. E. Bragg, H. E. Katz, Angew. Chem. Int. Ed. 2015, 54, 47824786.

[9] M. Irie, Chem. Rev. 2000, 100, 1685-1716.

[10] Y. Yokoyama, Chem. Rev. 2000, 100, 1717-1739.

[11] T. Mrozek, J. Daub, A. Ajayaghosh, in Molecular Switches (Ed.: B. L. Feringa), Wiley-VCH, Weinheim, 2001, pp. 63-106.

[12] F. Mançois, J.-L. Pozzo, J. Pan, F. Adamietz, V. Rodriguez, L. Ducasse, F. Castet, A. Plaquet, B. Champagne, Chem. Eur. J. 2009, 15, 2560-2571.

[13] F. Castet, V. Rodriguez, J.-L. Pozzo, L. Ducasse, A. Plaquet, B. Champagne, Acc. Chem. Res. 2013, 46, 2656-2665.

[14] R. Klajn, Chem. Soc. Rev. 2014, 43, 148-184.

[15] M. Irie, T. Fukaminato, K. Matsuda, S. Kobatake, Chem. Rev. 2014, 114, 12174-12277.

[16] P. A. Liddell, G. Kodis, A. L. Moore, T. A. Moore, D. Gust, J. Am. Chem. Soc. 2002, 124, 7668-7669.

[17] D. Higelin, H. Sixl, Chem. Phys. 1983, 77, 391-400.

[18] E. Hadjoudis, I. M. Mavridis, Chem. Soc. Rev. 2004, 33, 579-588.

[19] Y. Manolova, H. Marciniak, S. Tschierlei, F. Fennel, F. S. Kamounah, S. Lochbrunner, L. Antonov, Phys. Chem. Chem. Phys. 2017, 19, 7316-7325.

[20] S.-Z. Pu, Q. Sun, C.-B. Fan, R.-J. Wang, G. Liu, J. Mater. Chem. C 2016, 4, 3075-3093.

[21] S. O. Smith, Annu. Rev. Biophys. 2010, 39, 309-328. 
[22] R. W. Schoenlein, L. A. Peteanu, R. A. Mathies, C. V. Shank, Science 1991, 254, 412-415.

[23] B. L. Feringa, Angew. Chem. Int. Ed. 2017, 56, 11060-11078.

[24] D. Bléger, S. Hecht, Angew. Chem. Int. Ed. 2015, 54, 11338-11349.

[25] H. Ottosson, Nat. Chem. 2012, 4, 969-971.

[26] M. Rosenberg, C. Dahlstrand, K. Kilså, H. Ottosson, Chem. Rev. 2014, 114, 5379-5425.

[27] B. Oruganti, C. Fang, B. Durbeej, Phys. Chem. Chem. Phys. 2015, 17, 21740-21751.

[28] B. Oruganti, J. Wang, B. Durbeej, ChemPhysChem 2016, 17, 3399-3408.

[29] J. Wang, B. Oruganti, B. Durbeej, Phys. Chem. Chem. Phys. 2017, 19, 6952-6956.

[30] B. Oruganti, J. Wang, B. Durbeej, Org. Lett. 2017, 19, 4818-4821.

[31] B. Oruganti, J. Wang, B. Durbeej, Int. J. Quantum Chem. 2018, 118, e25405.

[32] L. Gutiérrez-Arzaluz, F. Cortés-Guzmán, T. Rocha-Rinza, J. Peón, Phys. Chem. Chem. Phys. 2015, 17, 31608-31612.

[33] M. J. S. Dewar, Tetrahedron 1966, 22 (Suppl. 8), 75-92.

[34] H. E. Zimmerman, J. Am. Chem. Soc. 1966, 88, 1564-1565.

[35] N. C. Baird, J. Am. Chem. Soc. 1972, 94, 4941-4948.

[36] V. Gogonea, P. v. R. Schleyer, P. R. Schreiner, Angew. Chem. Int. Ed. 1998, 37, 1945-1948.

[37] M. Garavelli, F. Bernardi, A. Cembran, O. Castaño, L. M. Frutos, M. Merchán, M. Olivucci, J. Am. Chem. Soc. 2002, 124, 13770-13789.

[38] H. Möllerstedt, M. C. Piqueras, R. Crespo, H. Ottosson, J. Am. Chem. Soc. 2004, 126, 1393813939.

[39] H. Ottosson, K. Kilså, K. Chajara, M. C. Piqueras, R. Crespo, H. Kato, D. Muthas, Chem. Eur. J. 2007, 13, 6998-7005.

[40] P. B. Karadakov, J. Phys. Chem. A 2008, 112, 7303-7309.

[41] P. B. Karadakov, J. Phys. Chem. A 2008, 112, 12707-12713.

[42] F. Feixas, J. Vandenbussche, P. Bultinck, E. Matito, M. Solà, Phys. Chem. Chem. Phys. 2011, 13, 20690-20703.

[43] K. Jorner, R. Emanuelsson, C. Dahlstrand, H. Tong, A. V. Denisova, H. Ottosson, Chem. Eur. J. 2014, 20, 9295-9303.

[44] P. B. Karadakov, P. Hearnshaw, K. E. Horner, J. Org. Chem. 2016, 81, 11346-11352.

[45] Y. M. Sung, J. Oh, W. Kim, H. Mori, A. Osuka, D. Kim, J. Am. Chem. Soc. 2015, 137, 1185611859. 
[46] Y. M. Sung, M.-C. Yoon, J. M. Lim, H. Rath, K. Naoda, A. Osuka, D. Kim, Nat. Chem. 2015, 7, 418-422.

[47] Y. M. Sung, J. Oh, K. Naoda, T. Lee, W. Kim, M. Lim, A. Osuka, D. Kim, Angew. Chem. Int. Ed. 2016, 55, 11930-11934.

[48] M. Hada, S. Saito, S. Tanaka, R. Sato, M. Yoshimura, K. Mouri, K. Matsuo, S. Yamaguchi, M. Hara, Y. Hayashi, F. Röhricht, R. Herges, Y. Shigeta, K. Onda, R. J. D. Miller, J. Am. Chem. Soc. 2017, 139, 15792-15800.

[49] H. Löfås, B. O. Jahn, J. Wärnå, R. Emanuelsson, R. Ahuja, A. Grigoriev, H. Ottosson, Faraday Discuss. 2014, 174, 105-124.

[50] R. K. Mohamed, S. Mondal, K. Jorner, T. F. Delgado, V. V. Lobodin, H. Ottosson, I. V. Alabugin, J. Am. Chem. Soc. 2015, 137, 15441-15450.

[51] R. Papadakis, H. Li, J. Bergman, A. Lundstedt, K. Jorner, R. Ayub, S. Haldar, B. O. Jahn, A. Denisova, B. Zietz, R. Lindh, B. Sanyal, H. Grennberg, K. Leifer, H. Ottosson, Nat. Commun. 2016, 7, 12962.

[52] N. Nishina, T. Mutai, J. Aihara, J. Phys. Chem. A 2017, 121, 151-161.

[53] J. Aihara, Bull. Chem. Soc. Jpn. 1978, 51, 1788-1792.

[54] S. Gozem, H. L. Luk, I. Schapiro, M. Olivucci, Chem. Rev. 2017, 117, 13502-13565.

[55] B. O. Roos, P. R. Taylor, P. E. M. Siegbahn, Chem. Phys. 1980, 48, 157-173.

[56] K. Andersson, P.-Å. Malmqvist, B. O. Roos, J. Chem. Phys. 1992, 96, 1218-1226.

[57] G. Groenhof, M. Bouxin-Cademartory, B. Hess, S. P. de Visser, H. J. C. Berendsen, M. Olivucci, A. E. Mark, M. A. Robb, J. Am. Chem. Soc. 2004, 126, 4228-4233.

[58] M. Barbatti, WIREs Comput. Mol. Sci. 2011, 1, 620-633.

[59] S. Mai, P. Marquetand, L. González, Int. J. Quantum Chem. 2015, 115, 1215-1231.

[60] I. F. Galván, M. G. Delcey, T. B. Pedersen, F. Aquilante, R. Lindh, J. Chem. Theory Comput. 2016, 12, 3636-3653.

[61] F. Aquilante, J. Autschbach, R. K. Carlson, L. F. Chibotaru, M. G. Delcey, L. De Vico, I. F. Galván, N. Ferré, L. M. Frutos, L. Gagliardi, M. Garavelli, A. Giussani, C. E. Hoyer, G. L. Manni, H. Lischka, D. Ma, P.-Å. Malmqvist, T. Müller, A. Nenov, M. Olivucci, T. B. Pedersen, D. Peng, F. Plasser, B. Pritchard, M. Reiher, I. Rivalta, I. Schapiro, J. Segarra-Martí, M. Stenrup, D. G. Truhlar, L. Ungur, A. Valentini, S. Vancoillie, V. Veryazov, V. P. Vysotskiy, O. Weingart, F. Zapata, R. Lindh, J. Comput. Chem. 2016, 37, 506-541. 
[62] S. Noorizadeh, E. Shakerzadeh, Phys. Chem. Chem. Phys. 2010, 12, 4742-4749.

[63] T. M. Krygowski, J. Chem. Inf. Comput. Sci. 1993, 33, 70-78.

[64] T. M. Krygowski, M. K. Cyrański, Chem. Rev. 2001, 101, 1385-1419.

[65] P. v. R. Schleyer, C. Maerker, A. Dransfeld, H. Jiao, N. J. R. v. E. Hommes, J. Am. Chem. Soc. 1996, 118, 6317-6318.

[66] R. F. W. Bader, Chem. Rev. 1991, 91, 893-928.

[67] C. E. Shannon, Bell Syst. Tech. J. 1948, 27, 379-423.

[68] A. Nikiforov, J. A. Gamez, W. Thiel, M. Filatov, J. Phys. Chem. Lett. 2016, 7, 105-110.

[69] M. Filatov, M. Olivucci, J. Org. Chem. 2014, 79, 3587-3600.

[70] P.-Å. Malmqvist, B. O. Roos, Chem. Phys. Lett. 1989, 155, 189-194.

[71] P. Lazzeretti, Phys. Chem. Chem. Phys. 2004, 6, 217-223.

[72] Z. Chen, C. S. Wannere, C. Corminboeuf, R. Puchta, P. v. R. Schleyer, Chem. Rev. 2005, 105, 3842-3888.

[73] M. K. Cyrañski, T. M. Krygowski, A. R. Katritzky, P. v. R. Schleyer, J. Org. Chem. 2002, 67, 1333-1338.

[74] P. Bultinck, Faraday Discuss. 2007, 135, 347-365.

[75] F. Feixas, E. Matito, J. Poater, M. Solà, J. Phys. Chem. A 2007, 111, 4513-4521.

[76] I. V. Omelchenko, O. V. Shishkin, L. Gorb, J. Leszczynski, S. Fias, P. Bultinck, Phys. Chem. Chem. Phys. 2011, 13, 20536-20548.

[77] E. Matito, J. Poater, M. Duran, M. Solà, J. Mol. Struct: THEOCHEM 2005, 727, 165-171.

[78] W. Rodríguez-Córdoba, J. S. Zugazagoitia, E. Collado-Fregoso, J. Peon, J. Phys. Chem. A 2007, 111, 6241-6247.

[79] J. H. Chong, M. Sauer, B. O. Patrick, M. J. MacLachlan, Org. Lett. 2003, 5, 3823-3826.

[80] A. D. Boese, N. C. Handy, J. Chem. Phys. 2002, 116, 9559-9569.

[81] E. I. Sánchez-Flores, R. Chávez-Calvillo, T. A. Keith, G. Cuevas, T. Rocha-Rinza, F. CortésGuzmán, J. Comput. Chem. 2014, 35, 820-828.

[82] M. García-Revilla, J. Hernández-Trujillo, Phys. Chem. Chem. Phys. 2009, 11, 8425-8432.

[83] C. García-Iriepa, M. Marazzi, L. M. Frutos, D. Sampedro, RSC Adv. 2013, 3, 6241-6266.

[84] A. Perrier, S. Aloise, M. Olivucci, D. Jacquemin, J. Phys. Chem. Lett. 2013, 4, 2190-2196.

[85] M. Cacciarini, A. B. Skov, M. Jevric, A. S. Hansen, J. Elm, H. G. Kjaergaard, K. V. Mikkelsen, M. B. Nielsen, Chem. Eur. J. 2015, 21, 7454-7461. 
[86] M. Paolino, M. Gueye, E. Pieri, M. Manathunga, S. Fusi, A. Cappelli, L. Latterini, D. Pannacci, M. Filatov, J. Léonard, M. Olivucci, J. Am. Chem. Soc. 2016, 138, 9807-9825.

[87] S. T. Olsen, M. B. Nielsen, T. Hansen, M. A. Ratner, K. V. Mikkelsen, ChemPhysChem 2017, 18, 1517-1525. 


\section{Biography}

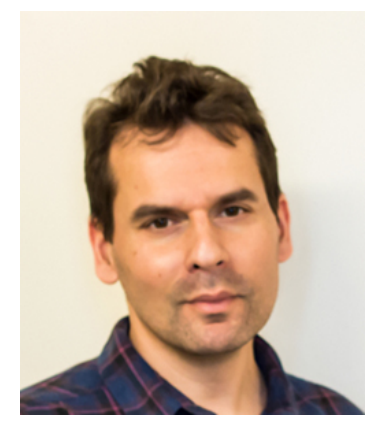

Bo Durbeej received his PhD from Uppsala University in 2004. Following postdoctoral work at the University of Sydney and the University of Siena, he then held a position at the Swedish Defence Research Agency before joining (in 2010) Linköping University, where he was promoted to Associate Professor in 2014. His recent research interests include molecular motors and switches, photosensory proteins and enzyme catalysis. 
Graphical suggestion for full-page picture
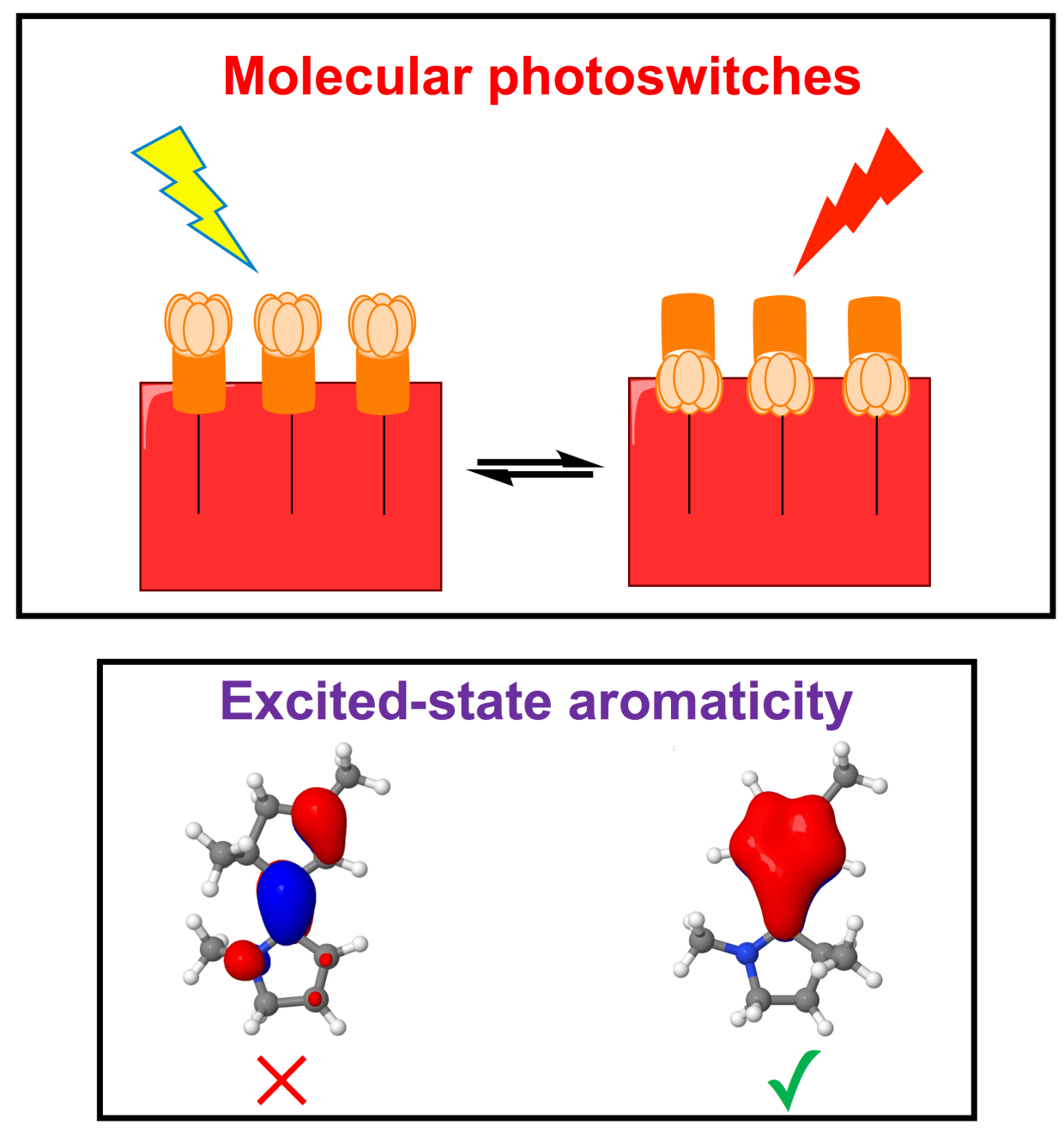

Quantum chemistry
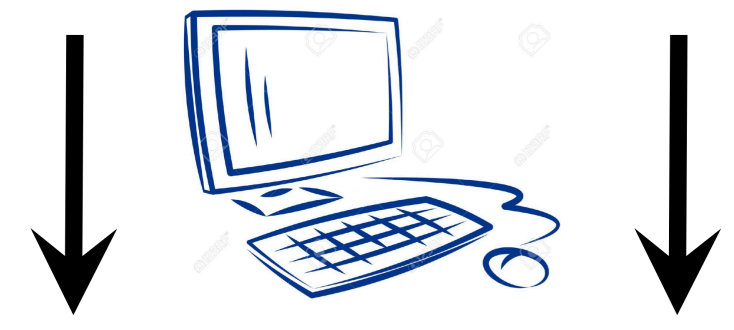

$$
\begin{array}{llrl}
\phi & =\sim 0.40 & \phi & =\sim 0.75 \\
\tau & =\sim 450 \mathrm{fs} & \tau & =\sim 200 \mathrm{fs}
\end{array}
$$




\section{Graphical abstract / Table of contents}

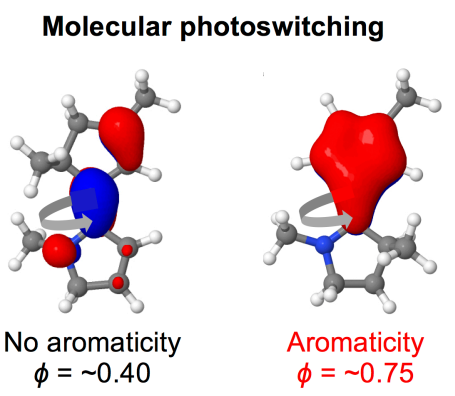

High quantum yields and fast response times are desirable features of molecular photoswitches in optoelectronics. The switches are often based on $E / Z$ photoisomerization or photoinduced $\mathrm{H}$ atom/proton transfer reactions, whereby it is of interest to develop new strategies to facilitate such reactions as much as possible. This minireview describes how recent computational studies have found that the concept of excited-state aromaticity has a key role to play for these endeavors. 